\title{
Estudios fisico-químicos de miscelas de aceites vegetales XIV. Volumen molar, refracción molar y viscosidad de disoluciones de estearato de metilo en ciclohexano, hexano, tetracloroetileno o tricloroetileno.
}

\author{
Por M.: J. Muñoz Cueto (1), M. Galán Vallejo (1) y V. Flores Luque (2).
}

(1) Departamento de Ingeniería Química. Universidad de Cádiz. Apdo. n. ${ }^{\&} 40$ Puerto Real (Cádiz) 11510. (2) Departamento de Ingenieria Química. Universidad de Sevilla.

\section{RESUMEN}

Estudios fisico-químicos de miscelas de aceites vegetales XIV. Volumen molar, refracción molar y viscosidad de disoluciones de estearate de metilo en ciclohexano, hexano, tetracloroetileno o tricloroetlleno.

En todas las miscelas estudiadas los valores del volumen molar y de la refracción molar presentan variaciones lineales en función de la fracción molar de estearato de metilo.

Los valores de viscosidad de las miscelas estudiadas se ajustan a la ecuación de fluidez de Hildebrand modificada, siendo su parámetro " $\mathrm{V}_{0}$ " función lineal de la fracción molar de estearato de metilo.

En relación con estas tres caracteristicas, el comportamiento de las miscelas estudiadas no difiere significativamente del correspondiente a una mezcla "ideal" de dos liquidos orgánicos sin ionizar.

PALABRAS-CLAVE: Densidad - Estearato de metilo - Indice de refracción - Miscela - Viscosidad.

\section{SUMMARY}

Physico-chemical studies concerning miscelles of vegetable oils XIV. Molar volume, molar refraction and viscosity for methyl stearate solutions in cyclohexane, hexane, tetrachloroethylene or trichloroethylene.

In the miscelles studied, molar volume and molar refraction values show linear dependence with the molar fraction of methyl stearate.

Viscosity values fit Hildebrand's modified equation, where the " $V_{0}$ " parameter is a linear function of the molar fraction of methyl stearate.

Regarding with this three physico-chemical properties, the mixtures studied behave like ideal mixtures of two organiq liquids without ionization.

KEY-WORDS: Density - Methyl stearate - Miscella - Refraction index - Viscosity.

\section{INTRODUCCION}

En comunicaciones anteriores de esta serie (1) (2) (3), se ha estudiado el comportamiento de las miscelas de diferentes ésteres metílicos de cadena larga y disolventes orgánicos.

Con esta comunicación se completa el estudio de los ésteres metílicos, estudiando las miscelas de estearato de metilo en ciclohexano, hexano, tetraclo- roetileno o tricloroetileno, por cuanto la escasa solubilidad del estearato en los referidos disolventes hace pensar que no será posible estudiar ésteres metílicos de mayor longitud de cadena. En posteriores comunicaciones se abordará el estudio del efecto que ejerce sobre los valores de volumen molar, refracción molar y viscosidad el número de átomos de carbono del éster, así como el efecto de la temperatura.

\section{PARTE EXPERIMENTAL}

\subsection{Productos empleados.}

Ciclohexano.- Su densidad a $293 \mathrm{~K}$ es $0,7783 \mathrm{~g} /$ $\mathrm{cm}^{3}$; su peso molecular medio 84,2 y su pureza, determinada por cromatografía gaseosa, del $99,7 \%$.

Hexano.- Su densidad a $293 \mathrm{~K}$ es $0,6612 \mathrm{~g} / \mathrm{cm}^{3}$; su peso molecular medio 86,2 y su pureza, determinada por cromatografía gaseosa, del 98,7\%.

Tetracloroetileno.- Su densidad a $293 \mathrm{~K}$ es 1,6243 $\mathrm{g} / \mathrm{cm}^{3}$, su peso molecular medio 165,8 y su pureza, determinada por cromatografía gaseosa, del $99 \%$.

Tricloroetileno.- Su densidad a $293 \mathrm{~K}$ es 1,4643 $\mathrm{g} / \mathrm{cm}^{3}$; su peso molecular medio 131,4 y su pureza, determinada por cromatografía gaseosa, del 99,5\%.

Estearato de metilo.- Su composición expresada en porcentaje en peso es: palmitato de metilo $0,3 \%$, estearato de metilo 99,7. El peso molecular medio 298,5 , el punto de fusión $312 \mathrm{~K}$, y la densidad, índice de refracción y viscosidad a $313 \mathrm{~K}$ son, respectivamente, $0,8680 \mathrm{~g} / \mathrm{cm}^{3} ; 0,8497$ y $4,67 \mathrm{mPa}$.s.

\subsection{Preparación de las miscelas.}

Las miscelas se han preparado por pesada, realizándose las determinaciones de densidad, índice de refracción y viscosidad sobre aquellas que se presentan en forma de una sola fase líquida a la temperatura de estudio. 


\subsection{Aparatos utilizados.}

Baño termostático.- Se ha utilizado un baño "Hetofrig" que permite controlar la temperatura en el intervalo estudiado, con la precisión de $\pm 0,1 \mathrm{~K}$.

Picnómetros.- Se han utilizado picnómetros de 10 $\mathrm{cm}^{3}$, con un solo brazo y tapón esmerilado. Su calibrado se realizó con agua destilada.

Refractómetro.- Se ha utilizado un refractómetro termostatable tipo "Abbe" de la firma "Atago", con una amplitud de escala de 1,3000 a 1,7100.
Viscosímetro.- Se ha utilizado un viscosímetro de caída de bola, termostatable, de tipo "Hoeppler" de la firma "Haake".

\section{RESULTADOS EXPERIMENTALES}

Los resultados obtenidos se indican en las Tablas I a IV, correspondiendo a cada fracción molar y temperatura de arriba a abajo: volumen específico (centímetros cúbicos/gramo), índice de refracción y viscosidad dinámica (milipascales - segundo).

Tabla 1

Miscelas de estearato de metilo y ciclohexano.

\begin{tabular}{|c|c|c|c|c|c|c|c|}
\hline$x_{\text {éster }}$ & $283 \mathrm{~K}$ & $288 \mathrm{~K}$ & $293 \mathrm{~K}$ & $298 \mathrm{~K}$ & $303 \mathrm{~K}$ & $308 \mathrm{~K}$ & $313 \mathrm{~K}$ \\
\hline \multirow[t]{3}{*}{0.00} & 1.2706 & 1.2786 & 1.2848 & 1.2928 & 1.3002 & 1.3085 & 1.3161 \\
\hline & - & 1.4300 & 1.4280 & 1.4255 & 1.4230 & 1.4205 & 1.4175 \\
\hline & 1.26 & 1.14 & 1.07 & 0.99 & 0.93 & 0.86 & 0.81 \\
\hline \multirow[t]{3}{*}{0.05} & 1.2541 & 1.2606 & 1.2670 & 1.2741 & 1.2814 & 1.2888 & 1.2958 \\
\hline & - & 1.4315 & 1.4295 & 1.4270 & 1.4250 & 1.4230 & 1.4205 \\
\hline & 1.61 & 1.45 & 1.33 & 1.22 & 1.14 & 1.06 & 0.99 \\
\hline \multirow[t]{3}{*}{0.10} & - & 1.2467 & 1.2536 & 1.2601 & 1.2669 & 1.2737 & 1.2802 \\
\hline & - & 1.4330 & 1.4310 & 1.4285 & 1.4265 & 1.4245 & 1.4225 \\
\hline & - & 1.77 & 1.61 & 1.46 & 1.35 & 1.25 & 1.16 \\
\hline \multirow[t]{3}{*}{0.15} & - & - & 1.2400 & 1.2463 & 1.2521 & 1.2584 & 1.2647 \\
\hline & - & - & 1.4330 & 1.4310 & 1.4290 & 1.4270 & 1.4245 \\
\hline & - & - & 1.92 & 1.73 & 1.58 & $1.46^{\circ}$ & 1.34 \\
\hline \multirow[t]{3}{*}{0.20} & - & - & 1.2295 & 1.2355 & 1.2415 & 1.2473 & 1.2533 \\
\hline & - & - & 1.4345 & 1.4320 & 1.4300 & 1.4285 & 1.4255 \\
\hline & - & - & 2.24 & 2.03 & 1.86 & 1.71 & 1.58 \\
\hline \multirow[t]{3}{*}{0.25} & - & - & .1 .2215 & 1.2271 & 1.2320 & 1.2375 & 1.2434 \\
\hline & - & - & 1.4360 & 1.4335 & 1.4315 & 1.4300 & 1.4275 \\
\hline & - & - & 2.58 & 2.33 & 2.12 & 1.95 & 1.78 \\
\hline \multirow[t]{3}{*}{0.30} & - & - & - & - & 1.2235 & 1.2288 & 1.2346 \\
\hline & -. & - & - & - & 1.4330 & 1.4310 & 1.4290 \\
\hline & - & - & - & - & 2.45 & 2.20 & 2.02 \\
\hline
\end{tabular}

Las medidas que no se han podido realizar por presentar carácter bifásico las miscelas, se han marcado con un guión.

El último dígito que aparece para cada una de las medidas es aquel que se ve afectado por los límites de confianza del método experimental utilizado.

\section{DISCUSION DE LOS RESULTADOS.}

\subsection{Refracción especifica de las miscelas.}

Se ha estudiado la refracción específica de las miscelas, definida como (4):

$$
R_{v}=\left[\left(n^{2}-1\right) / n^{2}+2\right] \cdot v
$$

donde " $n$ " es el índice de refracción y " $v$ " es el volumen específico en centímetros cúbicos/gramo. Para cada

miscela, a una concentración dada, los valores de la refracción específica no varían significativamente con la temperatura, teniendo en cuenta que los límites de confianza estimados para una probabilidad del $95 \%$ son inferiores a $\pm 0,0005$.

4.2. Volumen molar y refracción molar de las miscelas.

En base a los datos experimentales de volumen específico $\theta$ índice de refracción se han calculado los valores de volumen molar y refracción molar de las miscelas a diferentes temperaturas.

Para cada tipo de miscela y cada temperatura se encuentra una regresión lineal de las propiedades molares referidas frente a la fracción molar de éste 
Tabla II

Miscelas de estearato de metilo y hexano.

\begin{tabular}{|c|c|c|c|c|c|c|}
\hline$x_{\text {éster }}$ & $288 \mathrm{~K}$ & $293 \mathrm{~K}$ & $298 \mathrm{~K}$ & $303 \mathrm{~K}$ & $308 \mathrm{~K}$ & $313 \mathrm{~K}$ \\
\hline \multirow[t]{3}{*}{0.00} & 1.5035 & 1.5124 & 1.5225 & 1.5335 & 1.5439 & 1.5547 \\
\hline & 1.3800 & 1.3775 & 1.3750 & 1.3725 & 1.3700 & 1.3665 \\
\hline & 0.34 & 0.32 & 0.31 & 0.29 & 0.28 & 0.27 \\
\hline \multirow[t]{3}{*}{0.05} & 1.4463 & 1.4554 & 1.4643 & 1.4732 & 1.4819 & 1.4903 \\
\hline & 1.3895 & 1.3870 & 1.3850 & 1.3825 & 1.3805 & 1.3780 \\
\hline & 0.54 & 0.51 & 0.48 & 0.46 & 0.44 & 0.42 \\
\hline \multirow[t]{3}{*}{0.10} & - & 1.4084 & 1.4164 & 1.4245 & 1.4327 & 1.4408 \\
\hline & - & 1.3960 & 1.3940 & 1.3915 & 1.3895 & 1.3875 \\
\hline & - & 0.72 & 0.67 & 0.63 & 0.59 & 0.55 \\
\hline \multirow[t]{3}{*}{0.15} & - & 1.3723 & 1.3801 & 1.3877 & 1.3955 & 1.4035 \\
\hline & - & 1.4020 & 1.4000 & 1.3980 & 1.3960 & 1.3940 \\
\hline & - & 0.92 & 0.85 & 0.79 & 0.74 & 0.69 \\
\hline \multirow[t]{3}{*}{0.20} & - & 1.3414 & 1.3479 & 1.3543 & 1.3610 & 1.3689 \\
\hline & - & 1.4080 & 1.4060 & 1.4040 & 1.4020 & 1.4000 \\
\hline & - & 1.17 & 1.08 & 0.99 & 0.92 & 0.85 \\
\hline \multirow[t]{3}{*}{0.25} & - & - & 1.3227 & 1.3284 & 1.3356 & 1.3428 \\
\hline & - & - & 1.4115 & 1.4095 & 1.4085 & 1.4060 \\
\hline & - & - & 1.22 & 1.13 & 1.05 & 0.98 \\
\hline \multirow[t]{3}{*}{0.30} & - & - & 1.3011 & 1.3065 & 1.3123 & 1.3186 \\
\hline & - & - & 1.4155 & 1.4140 & 1.4125 & 1.4105 \\
\hline & - & - & 1.41 & 1.30 & 1.21 & 1.12 \\
\hline
\end{tabular}

Tabla III

Miscelas de estearato de metilo y tetracloroetileno.

\begin{tabular}{|c|c|c|c|c|c|c|c|}
\hline$x_{\text {êster }}$ & $283 \mathrm{~K}$ & $288 \mathrm{~K}$ & $293 \mathrm{~K}$ & $298 \mathrm{~K}$ & $303 \mathrm{~K}$ & $308 \mathrm{~K}$ & $313 \mathrm{~K}$ \\
\hline \multirow[t]{3}{*}{0.00} & 0.6103 & 0.6133 & 0.6160 & 0.6191 & 0.6222 & 0.6253 & 0.6285 \\
\hline & - & 1.5085 & 1.5065 & 1.5045 & 1.5020 & 1.4995 & 1.4970 \\
\hline & 1.20 & 1.14 & 1.08 & 1.02 & 0.97 & 0.92 & 0.88 \\
\hline \multirow[t]{3}{*}{0.05} & 0.6596 & 0.6626 & 0.6660 & 0.6692 & 0.6723 & 0.6759 & 0.6779 \\
\hline & - & 1.4985 & 1.4965 & 1.4940 & 1.4915 & 1.4895 & 1.4870 \\
\hline & 1.76 & 1.64 & .1 .51 & 1.41 & .1 .33 & 1.24 & 1.17 \\
\hline \multirow[t]{3}{*}{0.10} & - & 0.7039 & 0.7068 & 0.7106 & 0.7138 & 0.7165 & 0.7193 \\
\hline & - & 1.4915 & 1.4890 & 1.4865 & 1.4840 & 1.4820 & 1.4800 \\
\hline & - & 1.93 & 1.80 & 1.65 & 1.55 & 1.47 & 1.39 \\
\hline \multirow[t]{3}{*}{0.15} & - & - & 0.7474 & 0.7509 & 0.7542 & 0.7572 & 0.7610 \\
\hline & - & - & 1.4845 & 1.4825 & 1.4800 & 1.4780 & 1.4755 \\
\hline & - & - & 1.95 & 1.80 & 1.68 & 1.58 & 1.47 \\
\hline \multirow[t]{3}{*}{0.20} & - & - & 0.7857 & 0.7902 & 0.7930 & 0.7956 & 0.7992 \\
\hline & - & - & 1.4775 & 1.4750 & 1.4730 & 1.4710 & 1.4690 \\
\hline & - & - & 2.40 & 2.14 & 2.01 & 1.89 & 1.76 \\
\hline \multirow[t]{3}{*}{0.25} & - & - & 0.8168 & 0.8208 & 0.8249 & 0.8289 & 0.8331 \\
\hline & - & - & 1.4740 & 1.4720 & 1.4700 & 1.4680 & 1.4660 \\
\hline & - & - & 2.91 & 2.62 & 2.36 & 2.16 & 1.97 \\
\hline \multirow[t]{3}{*}{0.30} & - & - & - & 0.8565 & 0.8604 & 0.8641 & 0.8680 \\
\hline & - & - & - & 1.4670 & 1.4650 & 1.4635 & 1.4615 \\
\hline & -. & - & - & 2.93 & 2.63 & 2.40 & 2.20 \\
\hline
\end{tabular}


Tabla IV

Miscelas de estearato de metilo y tricloroetileno

\begin{tabular}{|c|c|c|c|c|c|c|c|}
\hline$x_{\text {éster }}$ & $283 \mathrm{~K}$ & $288 \mathrm{~K}$ & $293 \mathrm{~K}$ & $298 \mathrm{~K}$ & $303 \mathrm{~K}$ & $308 \mathrm{~K}$ & $313 \mathrm{~K}$ \\
\hline \multirow[t]{3}{*}{0.00} & 0.6758 & 0.6793 & 0.6829 & 0.6868 & 0.6906 & 0.6946 & 0.6986 \\
\hline & - & 1.4315 & 1.4790 & 1.4760 & 1.4735 & 1.4710 & 1.4685 \\
\hline & 0.80 & 0.76 & 0.73 & 0.69 & 0.66 & 0.63 & 0.60 \\
\hline \multirow[t]{3}{*}{0.05} & 0.7293 & 0.7327 & 0.7365 & 0.7407 & 0.7444 & 0.7488 & 0.7532 \\
\hline & - & 1.4735 & 1.4715 & 1.4695 & 1.4665 & 1.4645 & 1.4615 \\
\hline & 0.89 & 0.85 & 0.81 & 0.77 & 0.74 & 0.71 & 0.68 \\
\hline \multirow[t]{3}{*}{0.10} & 0.7725 & 0.7764 & 0.7799 & 0.7841 & 0.7881 & 0.7926 & 0.7966 \\
\hline & - & 1.4705 & 1.4680 & 1.4655 & 1.4630 & 1.4605 & 1.4585 \\
\hline & 1.22 & 1.15 & 1.10 & 1.04 & 0.98 & 0.93 & 0.89 \\
\hline \multirow[t]{3}{*}{0.15} & - & - & 0.8191 & 0.8234 & 0.8275 & 0.8317 & 0.8360 \\
\hline & - & - & 1.4645 & 1.4625 & 1.4600 & 1.4580 & 1.4550 \\
\hline & - & - & 1.48 & 1.37 & 1.30 & 1.21 & 1.14 \\
\hline \multirow[t]{3}{*}{0.20} & - & - & 0.8568 & 0.8612 & 0.8656 & 0.8695 & 0.8742 \\
\hline & - & - & 1.4615 & 1.4595 & 1.4575 & 1.4550 & 1.4530 \\
\hline & - & - & 1.88 & 1.73 & 1.60 & 1.50 & 1.40 \\
\hline \multirow[t]{3}{*}{0.25} & - & - & 0.8867 & 0.8910 & 0.8958 & 0.8996 & 0.9034 \\
\hline & - & - & 1.4590 & 1.4570 & 1.4545 & 1.4525 & 1.4505 \\
\hline & - & - & 2.29 & 2.09 & 1.90 & 1.77 & 1.66 \\
\hline \multirow[t]{3}{*}{0.30} & - & - & - & 0.9167 & 0.9205 & 0.9244 & 0.9289 \\
\hline & - & - & - & 1.4555 & 1.4530 & 1.4515 & 1.4490 \\
\hline & - & - & - & 2.61 & 2.39 & 2.20 & 2.01 \\
\hline
\end{tabular}

en la miscela que presenta en todos los casos un valor de "porcentaje de varianza no justificada" (5) definido como: "VNJ" $=100 \cdot\left(1-r^{2}\right)(N-1) /(N-2)$, inferior a 0,024 ; donde " $r$ " es el coeficiente de correlación lineal y " $\mathrm{N}$ " es el número de pares de datos utilizados para calcularlo.

Estas regresiones pueden expresarse de la forma siguiente:

$$
P_{M}=P_{1}+\left(P_{2}-P_{1}\right) x_{\text {ester }}
$$

donde $P_{M}$ es el valor de la propiedad molar de la miscela, $x_{\text {ester }}$ es la fracción molar de estearato de metilo $y, \stackrel{P_{1}}{P}$ y $P_{2}$ son parámetros de la recta de regresión, que deben corresponder respectivamente a los valores de la propiedad molar para el disolvente y estearato de metilo que forman la miscela, admitiendo la hipótesis de que éstas se comporten como mezclas ideales de dos líquidos orgánicos sin ionizar.

En las tablas $\mathrm{V}$ y $\mathrm{VI}$ se recogen los valores de los parámetros $P_{1}$ y $P_{2}$ correspondientes a cada una de las miscelas expresados en centímetros cúbicos $/ \mathrm{mol}$. Asimismo, se indican los valores de las propiedades molares determinadas experimentalmente de los disolventes y del éster, cuyo error se estima en 0,1 $\mathrm{cm}^{3} / \mathrm{mol}$ para una probabilidad del $95 \%$.

Los errores de los valores de los parámetros $P_{1}$ y $P_{2}$ para una probabilidad del $95 \%$, expresados en centímetros cúbicos/mol, son:

\begin{tabular}{|c|c|}
\hline $\begin{array}{l}\text { Volumen } \\
\text { molar }\end{array}$ & $\begin{array}{l}\text { Refracción } \\
\text { molar }\end{array}$ \\
\hline $\mathbf{P}_{1} \mathbf{P}_{2}$ & $\mathbf{P}_{1} \quad \mathbf{P}_{2}$ \\
\hline
\end{tabular}

Estearato de metilo-ciclohexano

$0,2 \quad 1,2 \quad 0,1 \quad 0,4$

$\begin{array}{llll}0,3 & 2,0 & 0,1 & 0,7\end{array}$

Estearato de metilo-hexano

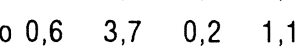

$\begin{array}{lllll}\text { Estearato de metilo-tricloroetileno } & 0,9 & 5,1 & 0,3 & 1,5\end{array}$

Del estudio de los valores correspondientes a los parámetros $P_{1}$ y $P_{2}$ y de los errores por los que se ven afectados, se deduce que el comportamiento de las miscelas estudiadas en relación al volumen molar y a la refracción molar no difiere del correspondiente a la hipótesis de mezclas ideales antes mencionada. Solamente se ha observado desviación del comportamiento ideal en las miscelas con hexano, si bien esta desviación es poco significativa y además sólo ha sido posible su consideración a $313 \mathrm{~K}$, pues a esa temperatura es a la que el estearato de metilo se presenta en estado líquido. En cualquier caso, las desviaciones observadas indican que se producen contracciones de volumen al mezclar los componentes de estas miscelas.

Los valores de volumen molar de las miscelas, expresados en centímetros cúbicos/mol, varian linealmente con la temperatura, expresada en $\mathrm{K}$, resultando valores de VNJ inferiores a 0,2 en todos los casos. 
Tabla V

Parámetros correspondientes a volúmenes molares $\left(\mathrm{cm}^{3} / \mathrm{mol}\right)$.

\begin{tabular}{|c|c|c|c|c|c|}
\hline TIPO DE MISCELA & & $298 \mathrm{~K}$ & $303 \mathrm{~K}$ & $308 \mathrm{~K}$ & $313 \mathrm{~K}$ \\
\hline \multirow{2}{*}{ Estearato de metilo-ciclohexano } & $\mathrm{P}_{1}$ & -- & 109,6 & 110,3 & 111,0 \\
\hline & $\mathrm{P}_{2}$ & -- & 350,0 & 351,0 & 352,4 \\
\hline \multirow{2}{*}{ Estearato de metilo-hexano } & $\mathrm{P}_{1}$ & 130,9 & 131,8 & 132,7 & 133,7 \\
\hline & $\mathrm{P}_{2}$ & 344,2 & 344,8 & 345,9 & 347,4 \\
\hline \multirow{2}{*}{ Estearato de metilo-tetracloroetileno } & $\mathrm{P}_{1}$ & 103,2 & 103,6 & 104,1 & 104,4 \\
\hline & $\mathrm{P}_{2}$ & 345,5 & 347,1 & 348,5 & 350,7 \\
\hline \multirow{2}{*}{ Estearato de metilo-tricloroetileno } & $\mathrm{P}_{1}$ & 91,0 & 91,5 & 92,1 & 92,7 \\
\hline & $\mathrm{P}_{2}$ & 343,5 & 344,9 & 345,8 & 347,1 \\
\hline \multicolumn{6}{|l|}{ VALORES EXPERIMENTALES } \\
\hline $\begin{array}{l}\text { Ciclohexano } \\
\text { Hexano } \\
\text { Tetracloroetileno } \\
\text { Tricloroetileno } \\
\text { Estearato de metilo }\end{array}$ & & $\begin{array}{c}108,8 \\
131,2 \\
102,7 \\
90,2 \\
--\end{array}$ & $\begin{array}{r}109,5 \\
132,2 \\
103,2 \\
90,7 \\
--\end{array}$ & $\begin{array}{r}110,2 \\
133,4 \\
103,7 \\
91,3 \\
--\end{array}$ & $\begin{array}{r}110,8 \\
134,0 \\
104,2 \\
91,8 \\
351,3\end{array}$ \\
\hline
\end{tabular}

Tabla VI

Parámetros correspondientes a las refracciones molares $\left(\mathrm{cm}^{3} / \mathrm{mol}\right)$

\section{TIPO DE MISCELA}

Estearato de metilo-ciclohexano

Estearato de metilo-hexano

Estearato de metilo-tetracloroetileno

Estearato de metilo-tricloroetileno

\section{VALORES EXPERIMENTALES}

\section{Ciclohexano}

Hexano

Tetracloroetileno

Tricloroetileno

Estearato de metilo

En cuanto a la refracción molar hay que indicar que, a tenor de los valores reflejados en la Tabla VI y de los errores por los que se ven afectados los parámetros en ella indicados, las miscelas de estearato de metilo con los disolventes estudiados no difieren significativamente en su comportamiento del correspondiente a la hipótesis de mezcla ideal.

\begin{tabular}{|c|c|c|c|c|}
\hline & $298 \mathrm{~K}$ & $303 \mathrm{~K}$ & $308 \mathrm{~K}$ & $313 \mathrm{~K}$ \\
\hline$P_{1}$ & -- & 27,9 & 27,9 & 27,9 \\
\hline$P_{2}$ & -- & 92,3 & 92,4 & 92,4 \\
\hline$P_{1}$ & 30,1 & 30,1 & 30,1 & 30,1 \\
\hline $\mathrm{P}_{2}$ & 92,8 & 92,9 & 93,1 & 93,1 \\
\hline $\mathrm{P}_{1}$ & 30,5 & 30,5 & 30,5 & 30,5 \\
\hline $\mathrm{P}_{2}$ & 91,5 & 91,7 & 91,8 & 92,0 \\
\hline $\mathrm{P}_{1}$ & 25,6 & 25,6 & 25,7 & 25,7 \\
\hline $\mathrm{P}_{2}$ & 91,0 & 91,1 & 91,1 & 91,1 \\
\hline & $\begin{array}{c}27,9 \\
30,0 \\
30,4 \\
25,4 \\
--\end{array}$ & $\begin{array}{c}27,9 \\
30,1 \\
30,4 \\
25,5 \\
--\end{array}$ & $\begin{array}{c}27,9 \\
30,1 \\
30,5 \\
25,5 \\
--\end{array}$ & $\begin{array}{l}27,9 \\
30,1 \\
30,5 \\
25,5 \\
92,0\end{array}$ \\
\hline
\end{tabular}

La interpretación de los resultados obtenidos para las miscelas estudiadas se ha realizado considerando el modelo del "retículo miscelar" reflejado en una comunicación anterior (2).

Con las limitaciones impuestas por la solubilidad del estearato de metilo en los distintos disolventes dentro del intervalo de temperaturas estudiado, se 
observan contracciones de volumen en el caso de las miscelas de palmitato de metilo con hexano que se atribuyen, como en el caso de las miscelas de miristato y palmitato de metilo, a un aumento en el grado de estructuración de la miscela debido a la existencia de interacciones intensas entre las moléculas de los dos componentes, como pueden ser fuerzas de Van de Waals, London, etc., y a la semejanza de estructura entre las moléculas que forman la miscela.

En el caso de las miscelas de palmitato de metilo preparadas con tricloroetileno, se observan contracciones de volumen que se atribuyen a interacciones debidas al momento dipolar del tricloroetileno.

En el caso de las miscelas con ciclohexano y tetracloroetileno, la falta de semejanza en dimensiones y estructura con las moléculas de éster de metilo, justifica el comportamiento ideal en lo referente a volúmenes molares parciales en la miscela.

Estos efectos observados no deben traer consigo alteración alguna en las estructuras electrónicas de las moléculas que forman las miscelas, como se deduce del comportamiento ideal de estas miscelas en lo referente a la refracción molar.

\subsection{Relación entre la viscosidad dinámica y el} volumen molar.

En la última comunicación de esta serie (3) se ha estudiado el ajuste de los valores experimentales obtenidos para las miscelas de palmitato de metilo con diferentes disolventes orgánicos a la ecuación de fluidez de Hildebrand modificada. Para las miscelas de estearato de metilo estudiadas, como ocurría en el caso de las referidas miscelas de palmitato, los intervalos de valores experimentales de volumen molar son relativamente reducidos, y es por ello que se ha realizado el ajuste de los valores experimentales obtenidos a la referida ecuación de fluidez de Hildebrand modificada de la forma:

$$
\varnothing=\left[\mathrm{B}_{r} /\left(\mathrm{V}_{r}-\mathrm{V}_{0}\right)\right] \cdot \mathrm{V}_{m}-\left(\mathrm{B}_{r} \cdot \mathrm{V}_{r} /\left(\mathrm{V}_{r}-\mathrm{V}_{0}\right)\right) / 2 /
$$

donde " $\varnothing$ " (milipascales $\times$ segundo) ${ }^{-1}$ es la fluidez (inversa de la viscosidad dinámica) y " $V_{m}$ " (centímetros cúbicos $/ \mathrm{mol}$ ) el volumen molar correspondiente a una misma temperatura, " $\mathrm{B}_{r}$ " (miliPascales - segundo) corresponde al valor de fluidez calculado aplicando la ecuación de Hildebrand $\varnothing=\mathrm{B}\left(\left(\mathrm{V}-\mathrm{V}_{0}\right) / \mathrm{V}_{0}\right)$ (6) a un valor de volumen molar designado por " $\mathrm{Vr}$ " próximo al intervalo experimental, y " $V_{0}$ " es el denominado volumen límite, su significado es el del volumen en el cual las moléculas contenidas en un mol están lo suficientemente próximas como para impedir la autodifusión y flujo viscoso sin que se anule el flujo plástico.

Los valores de " $\mathrm{V}$," elegidos son los mismos que en la comunicación anterior (3): $117 \mathrm{~cm}^{3} / \mathrm{mol}$ para las miscelas con ciclohexano; $135 \mathrm{~cm}^{3} / \mathrm{mol}$ para las miscelas con hexano y $100 \mathrm{~cm}^{3} / \mathrm{mol}$ para las miscelas con los disolventes clorados.

Los valores del parámetro "Br" (miliPascales - segundo) y sus errores para una probabilidad del $95 \%$ correspondientes a las miscelas estudiadas se encuentran en la Tabla VII junto con los valores de los parámetros " $V_{0}$ " (centímetros cúbicos $/ \mathrm{mol}$ ) y "B" (miliPascales - segundo) de la ecuación de Hildebrand y sus errores para una probabilidad del $95 \%$.

Tabla VII

Valores de los parámetros de la ecuación de Hildebrand y ecuación de Hildebrand modificada.

$$
\mathrm{V}_{0}: \mathrm{cm}^{3} / \mathrm{mol} \quad \mathrm{B}:(\mathrm{mPa} . \mathrm{s})^{-1} \quad \mathrm{~B}_{\mathrm{r}}:(\mathrm{mPa} . \mathrm{s})^{-1}
$$

\begin{tabular}{|c|c|c|c|c|c|c|c|c|c|c|c|c|}
\hline \multirow[b]{2}{*}{$x_{\text {éster }}$} & \multicolumn{3}{|c|}{$\begin{array}{l}\text { ESTEARATO DE METILO } \\
\text { CICLOHEXANO }\end{array}$} & \multicolumn{3}{|c|}{$\begin{array}{l}\text { ESTEARATO DE METILO } \\
\text { HEXANO }\end{array}$} & \multicolumn{3}{|c|}{$\begin{array}{l}\text { ESTEARATO DE METILO } \\
\text { TETRACLOROETILENO }\end{array}$} & \multicolumn{3}{|c|}{$\begin{array}{l}\text { ESTEARATO DE METILO } \\
\text { TRICLOROETILENO }\end{array}$} \\
\hline & $v_{0}$ & B & ${ }^{B_{r}}$ & $v_{0}$ & B & $B_{r}$ & $v_{0}$ & B & $B_{r}$ & $v_{0}$ & B & $\mathrm{B}_{\mathrm{r}}$ \\
\hline 0,00 & $100 \pm 6$ & $11,4 \pm 0,4$ & $1,93 \pm 0,14$ & $113 \pm 11$ & $20,3 \pm 1,5$ & $3,96 \pm 0,48$ & $93 \pm 3$ & $9,4 \pm 0,3$ & $0,71 \pm 0,03$ & $80 \pm 2$ & $11,3 \pm 0,5$ & $2.78 \pm 0.11$ \\
\hline 0,05 & $113 \pm 6$ & $10,9 \pm 0,5$ & $0,38 \pm 0,02$ & $127 \pm 10$ & $17,8 \pm 1,1$ & $1.12 \pm 0.14$ & $107 \pm 4$ & $9,0 \pm 0,2$ & $-0.59 \pm 0,02$ & $91 \pm 4$ & $9,4 \pm 0,4$ & $0,93 \pm 0,04$ \\
\hline 0,15 & $138 \pm 8$ & $10,7 \pm 0,5$ & $-1,63 \pm 0,13$ & $151 \pm 12$ & $14,7 \pm 0,9$ & $-1,56 \pm 0,12$ & $131 \pm 5$ & $8,6 \pm 0,2$ & $-2,03 \pm 0,08$ & $119 \pm 5$ & $8,8 \pm 0,3$ & $-1,40 \pm 0,06$ \\
\hline 0,20 & $149 \pm 9$ & $9,3 \pm 0,4$ & $-2,00 \pm 0,08$ & $162 \pm 12$ & $13,0 \pm 0,9$ & $-2,16 \pm 0,21$ & $144 \pm 5$ & $8,4 \pm 0,3$ & $-2.56 \pm 0.09$ & $133 \pm 6$ & $8,6 \pm 0,3$ & $-2,13 \pm 0,10$ \\
\hline 0.25 & $162 \pm 10$ & $9,4 \pm 0,4$ & $-2,61 \pm 0,18$ & $173 \pm 11$ & $12,2 \pm 0.8$ & $-2,69 \pm 0,28$ & $156 \pm 5$ & $8,2 \pm 0,2$ & $-2,94 \pm 0,10$ & $146 \pm 6$ & $8,4 \pm 0,3$ & $-2,65 \pm 0,11$ \\
\hline 0,30 & $174 \pm 11$ & $9,2 \pm 0,5$ & $-3,00 \pm 0,19$ & $184 \pm 13$ & $12,5 \pm 0,7$ & $-3,31 \pm 0,37$ & $169 \pm 6$ & $8,1 \pm 0,2$ & $-3,30 \pm 0,12$ & $159 \pm 7$ & $8.2 \pm 0,3$ & $-3,04 \pm 0,22$ \\
\hline
\end{tabular}




\section{ESTUDIO DEL PARAMETRO " $\mathrm{V}_{0}$ "}

En las diferentes miscelas de estearato de metilo estudiadas se ha encontrado una variación lineal de los valores del parámetro " $V_{0}$ " con la fracción molar de estearato de metilo en la miscela. Dicha variación puede expresarse de la forma:

$$
V_{0}=V_{01}+\left(V_{02}-V_{01}\right) x_{\text {oster }}
$$

donde " $V_{01}$ " y " $V_{02}$ " son parámetros de ajuste cuyos valores deben corresponder respectivamente al volumen límite del disolvente y del estearato de metilo, $y$ " $x_{\text {ester }}$ " es la fracción molar de estearato de metilo en la miscela. Los valores de VNJ son inferiores a 0,17 .

Los valores de los parámetros de la correlación, expresados en centímetros cúbicos/mol, junto con los errores por los que se ven afectados para una probabilidad del $95 \%$ son los siguientes:

\begin{tabular}{ll}
$V_{01}$ & $V_{\infty}$ \\
\hline
\end{tabular}

Estearato de metilo-ciclohexano

$99,5 \pm 0,5 \quad 272,5 \pm 2,0$

Estearato de metilo-hexano

$115,1 \pm 1,1 \quad 269,3 \pm 4,4$

Estearato de metilo-tetracloroetileno $92,9 \pm 1,0 \quad 270,6 \pm 3,8$

Estearato de metilo-tricloroetileno $76,7 \pm 2,1 \quad 275,2 \pm 9,1$

Hay que indicar que los valores del parámetro " $\mathrm{V}_{02}$ " corresponden a fracción molar de estearato de metilo igual a la unidad y debido a la insolubilidad de éste no es posible estudiar todo el intervalo de fracción molar, como se indica en las tablas de resultados experimentales.

Esto supone que no se dispone de datos experimentales suficientes, en el intervalo de temperatura objeto de estudio, que permitan estimar el valor del volumen límite " $V_{0}$ " correspondientes a las miscelas de fracción molar elevada (superiores a 0,3 ) de estearato de metilo, por lo cual hay que indicar que los valores de " $V_{01}$ " obtenidos comprenden, dentro de sus márgenes de error, a los valores de " $V_{0}$ " correspondiente al disolvente en cada caso, así como que los valores de " $V_{02}$ " obtenidos no difieren del valor del volumen molar del estearato de metilo a la temperatura del punto de fusión.
En consecuencia, se puede afirmar que las miscelas de estearato de metilo estudiadas no se apartan significativamente de la ley de aditividad respecto del volumen límite " $V_{0}$ " de la ecuación de Hildebrand.

\section{ESTUDIO DEL PARAMETRO "B,"}

Al comparar los valores obtenidos para los parámetros " $B$," y " $B$ " se observa que los valores de "B," presentan una disminución regular con la fracción molar de estearato de metilo en la miscela, mientras que los valores de " $B$ " no.

Referente a los valores de "B" se observa que, para la misma fracción molar de éster metílico, los valores de este parámetro no difieren significativamente entre sí cuando se trata de miscelas de miristato (2), palmitato (3) o estearato de metilo.

\section{CONSIDERACIONES FINALES}

1. - Para cada tipo de miscela estudiada, a una concentración dada, los valores de refracción específica no varian de forma significativa con la temperatura.

2.- Los valores de volumen molar y de refracción molar de las miscelas cumplen la ley de aditividad de mezclas, existiendo desviaciones poco significativas de dicha ley en el caso de las miscelas con hexano y tricloroetileno.

3.- Las miscelas estudiadas se ajustan a la ecuación de fluidez de Hildebrand modificada en la forma:

$$
\varnothing=\left[B_{r} /\left(V_{r}-V_{0}\right)\right] \cdot V_{m}-\left\{\left[B_{r} \cdot V_{r} /\left(V_{r}-V_{0}\right)\right]-1\right\}
$$
encontrándose relaciones lineales de los valores de " $V_{0}$ " y la fracción molar de estearato de metilo, así como una variación regular de " $B$," con la fracción molar de estearato en el sentido de disminuir su valor al aumentar la fracción molar.

4.- El coeficiente de dilatación molar volumétrica a presión constante no varía para cada una de las miscelas dentro del intervalo de temperatura estudiado.

5.- En relación a las propiedades estudiadas, el comportamiento de las miscelas de estearato de metilo con ciclohexano, hexano, tetracloroetileno o tricloroetileno, se aproxima al de las mezclas binarias ideales 
BIBLIOGRAFIA

1. Flores Luque, V.; Gómez Herrera, C.; Galán Vallejo, M. y Sancho González, A.M." .- "Estudios fisico-químicos de miscelas de aceites vegetales. VIII. Volumen molar, refracción molar y viscosidad de las disoluciones de laurato de metilo en hexano, ciclohexano, tetracloroetileno o tricloroetileno" .- Grasas y Aceites 34 (1983) 95100.

2. Muñoz Cueto, M.'J.; Galán Vallejo, M. y Flores Luque, V.-"Estudios fisico-quimicos de miscelas de aceites vegetales. XII. Volumen molar, refracción molar y viscosidad de disoluciones de miristato de metilo en ciclohexano, hexano, tetracloroetileno o tricloroetileno" .- Grasas y Aceites 41 (1990) 59-68.

3. Muñoz Cueto, M.'J.; Galán Vallejo, M.; Martinez Moreno, J. M. y Flores Luque, V.- "Estudios fisico-químicos de miscelas de aceites vegetales. XIII. Volumen molar, refracción molar y viscosidad de disoluciones de palmitato de metilo en ciclohexano hexano, tetracloroetileno o tricloroetileno".- Grasas y Aceites 41 (1990) 324-331.

4. Glasstone, S.- "Tratado de Quimica Fisica".- Ed. Aguilar, Madrid, 1968.

5. Flores Luque, V.; Cabrera Martín, J. y Gómez Herrera, C.- "Estudio fisico-químico de miscelas de aceites vegetales. $X$. Relación entre fluidez y volumen específico en miscelas binarias de un aceite de girasol o de soja crudo y tricloroetileno o percloroetileno".Grasas y Aceites 34 (1983) $380-385$.

(Recibido: Abril 1990) 